\title{
Primary Germ Cell Tumour of Testis with Sarcomatous Changes: A Unique Case Report
}

\author{
Authors \\ Ashutosh Silodia', Muktesh Khandare ${ }^{2}$, Piyush Kumar Panchariya ${ }^{3}$, \\ Dhananjay Sharma ${ }^{4}$ \\ ${ }^{1,3,4}$ Department of Surgery, NSCB Medical College, Jabalpur, India \\ ${ }^{2}$ Department of Pathology, NSCB Medical College, Jabalpur, India \\ Corresponding Author \\ Ashutosh Silodia \\ Department of Surgery, NSCB Medical College Jabalpur \\ Email: silodiaashu@gmail.com, Contact No.9826178486
}

\begin{abstract}
This paper reports a unique case of undifferentiated germ cell tumour with sarcomatous changes. The lack of differentiation into any specific germ cell type along with microscopic spindle and plump cells with immunohistochemistry positive for desmin and high ki67index but negative for PLAP along with normal markers of testicular malignancy apart from increased LDH marks the presence of undifferentiated germ cell tumour with sarcomatous changes and a high proliferative index. Although the patient had the localised disease at the time of presentation, the prognosis of the disease expected to be guarded and needs atailored and individualised approach for a better management and control of disease.

Keywords-Germ Cell Tumour, Sarcoma, Undifferentiated testicular tumours, Testis, Rhabdomyoblastoma, Testicular Tumour Markers.
\end{abstract}

\section{Introduction}

Testicular tumours account for $1 \%$ of all malignant tumours. $99 \%$ of testicular tumours are malignant. The life time prevalence is $0.2 \%$. Histologically testicular tumours may be classified as germ cell tumours $95 \%$ and non germ cell tumours $5 \%$. Further germ cell tumours may be subclassified as seminomatous, embryonal cell carcinoma, teratoma, choriocarcinoma, mixed cell type or carcinoma in situ. These histologic type guides treatment plan. Seminomas are sensitive to radiotherapy while teratomas are less sensitive to radiotherapy. Non seminomatous germ cell tumours respond to cisplatin based chemotherapy.
Sarcomatous transformation has been described as a component of germ cell tumours specially in cases of teratoma and yolk sac tumours and also in ovarian germ cell tumours. Once sarcoma develops, chances of their metastasis increase. Sarcomatous changes have been reported after prior chemotherapy as metastasis or recurrence at primary site, subjecting them to be chemoresistant.

Most of the cases reported previously developed sarcomatous changes either in secondaries or recurrences while only few primary cases with sarcomatous changes have been reported. 


\section{Case Report}

A 22 year old young man resident of Pipri, dist Singruali, Madhya Pradesh, India labour by occupation presented with chief complaints of swelling over right testicle for 3 months. The patient was apparently alright 3 months back when he noticed a swelling over his right testicle which was insidious in onset, of a size of about 3 by $2 \mathrm{~cm}$ to start with and progressed to a size of about 5 by 5 $\mathrm{cm}$ over a period of 3 months. The swelling had been painless, not changing in size with change in posture, not associated with any inguinal swelling with no history of any fever, vomiting, burning micturition, any mass in abdomen, lower limb swelling or distended veins over lower limb, passing flatus motion normally. There was no history of cough, hemoptysis, jaundice or weight loss. There was no history of any trauma. Testis were present in scrotum since birth. There was no significant medical history. No significant past history was there. Patient was vegetarian by diet and no history of any addiction. Bladder bowel, sleep, apetite were normal.

Upon examination, patient was conscious and well oriented to time, place and person, average built, mildly anaemic, no icterus/ cyanosis/ lower limb oedema. Systemic examination of CNS, CVS and respiratory system was within normal limit. On per abdomen examination, abdomen not distended, no distended veins and bowel sound present with no palpable organomegaly or any lump.

On testis examination, upon inspection, there was a right sided globular right scrotal swelling of size about 7 by $4 \mathrm{~cm}$, overlying skin normal in colour and texture, scrotal rugosities on right side diminished relative to left side. Penis was not deviated. No visible cough impulse in inguinal region. Contralateral testis appeared to be normal in shape, size and location. On palpation findings of inspection were confirmed. The right scrotal globular swelling of size 7 by $4 \mathrm{~cm}$, non tender to touch, hard in consistency, getting above possible, reduced sensations to touch and pressure, overlying skin free from swelling, non reducible non fluctuant, no cord involvement, no plapable cough impulse, non transilluminant. Contralateral testis was normal in shape size consistency and sensations. No palpable inguinal, abdominal or supraclavicular lymph nodes.

On USG, there was a hypoechoic mass in right testis with areas of necrosis in between with increased vascularity and no secondary hydrocele. Cord was normal and not involved. The opposite testis was normal.

On serum marker analysis, Sr AFP $1.2 \mathrm{ng} / \mathrm{ml}$ (Ref < $3.5 \mathrm{ng} / \mathrm{ml}$ ), Sr Beta HCG 0.3 Miu/ml (Ref < 5.3), and Sr LDH 297 IU/L (Ref 125-220)

On surgical exploration via high inguinal approach, testis delivered to the wound, it was found to be a hard mass involving whole of the testis with some necrotic areas in between no cord involvement. The cord cut at deep inguinal ring and specimen delivered en bloc and sent for HPR evaluation.

Findings of HPR as per the pathology reports were "grossly normal testis not seen, it was replaced by tumour along with $4.5 \mathrm{~cm}$ of spermatic cord. The dimension of main tumour mass was 12 × 8 x $5 \mathrm{~cm}$ ". Microscopically section from left testicular mass (Tumour) shows microcystic spaces lined by tumour cells, which revealed round to oval, irregularly shaped nuclei, inconspicuous nucleoli and indistinct to scanty amount of cytoplasm. Tumour cells admixed with abundant multinucleated giant cells, bizarre spindle cells and plump cells with rhabdomyoblastic differentiation. Spindle cells having spindle shaped, marked pleomorphic nuclei, irregular distributed chromatin and abundant amount of eosinophilic cytoplasm with cross striations. Tumor was involving tunica albuginea and tunica vaginalis, however epididymis not appreciated and surgical resected margin of spermatic cord were free of tumors.

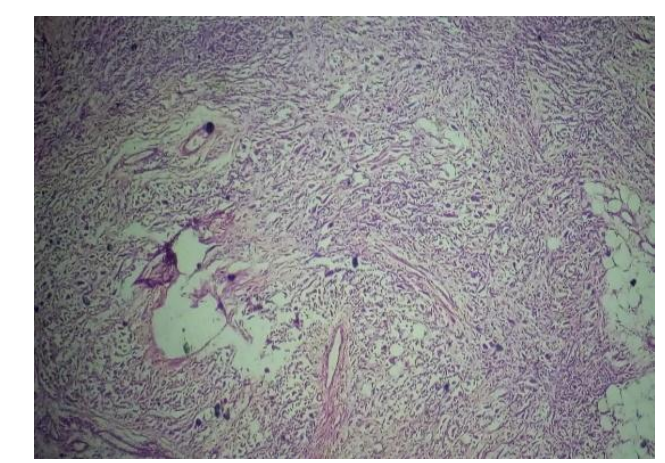



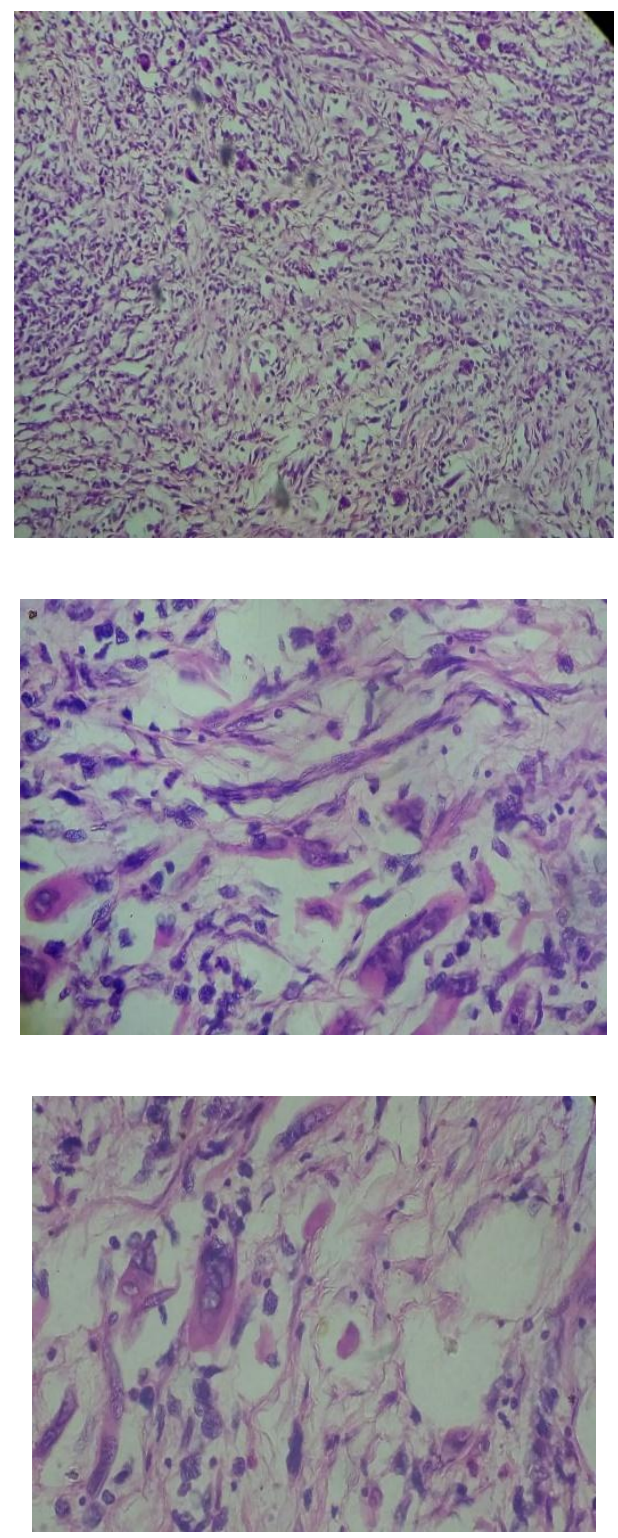

On the microscopic features the diagnosis was "Testicular Germ cell Tumour with Sarcomatous changes". Staging of tumour on the basis of TNM was pT1 and was advised for immunohistochemistry (PLAP, OCT-3/4, Desmin, myogenin, CK(AE1/AE3)).

Upon IHC tumour came to be strongly postitive for desmin in plump and spindle striated component with high ki67 proliferation index but negative for PLAP and myogenin negative. This was unequivocally in favour of presence of rhabdomyosarcoma.

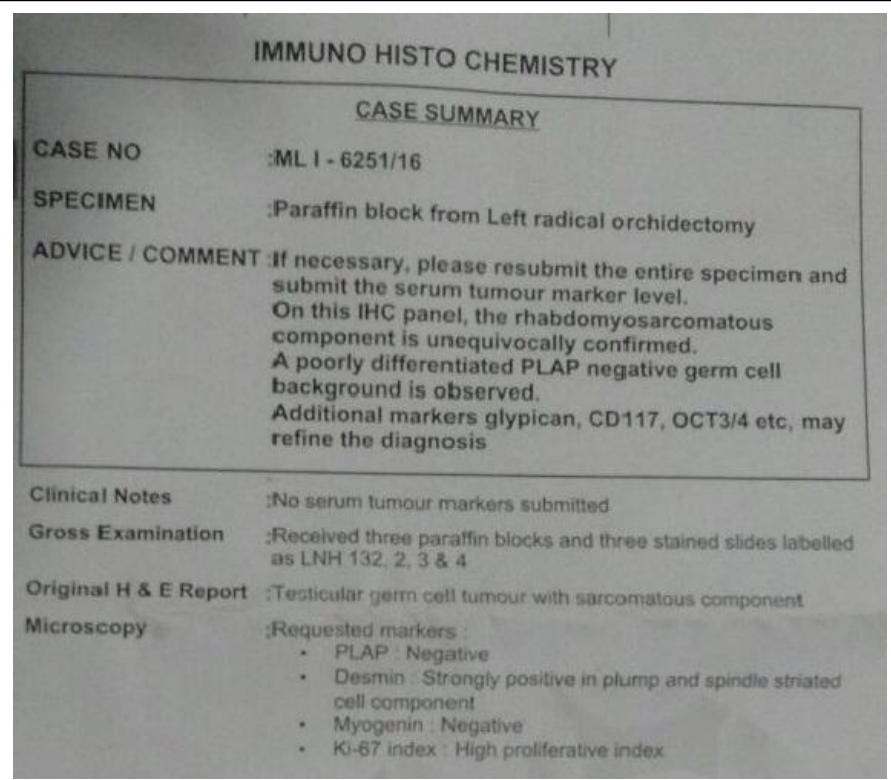

\section{Discussion}

Testicular tumours chiefly comprise of carcinomas of germ cell or non germ cell origins. Primary testicular sarcoma or germ cell tumours with sarcomatoid changes have been rarer events in the diagnosis and have a unique negative inference in the prognosis of testicular malignancies. Previous studies have reported presence of sarcomatous changes uniquely in cases of non seminomatous germ cell tumours like teratomas and yolk sac tumours but rarer in seminomas with the exception of spermatocytic variant of seminoma. Sarcomas have also been found in cases of recurrences and in distant metastasis following testicular carcinomas.

Here in our case tumour was not distinctly differentiated in any of the varieties of germ cell tumours but positively having a sarcomatoid component on histopathological analysis and also supported with the results of immunohistochemistry (desmin postive). The most common sarcomatous elements found in testicular malignancies has been rhabdomyoblastic in origin and similar sarcomatous origin was also found in our case too. The case is unique in its own as typical differentiation into specific variant of germ cell tumour was not clearly distinct along with normal tumour marker apart from LDH suggesting the load of tumour. The tumour also showed high ki67 index for proliferation corroborating with a view of rapidly progressing nature of the tumour with sarcomatoid 
differentiation. Further analysis with cytogenetic analysis and markers like OCT 4/ OCT 6 were needed for further conclusions.

The presence of sarcomatoid differentiation is significant with respect to the point of prognosis as it carries an extremely poor prognosis with low 5 year survival rates. This may account to the fact that chemotherapy against a particular cell line targets and kills that cell line and allowing the other one to survive and proliferate. No protocol guidelines for management have been deviced yet for management of such testicular malignancies. Therapy needs to be individualised and tailored approach should be adopted in each case with special reference to target sarcomatoid cells for a good control of disease and prolonging survival of patients.

\section{Conclusion}

Although rarer, either primary sarcomas or sarcomatous differentiation in germ cell tumours of testis significantly affect the prognosis and treatment of the patient. If sarcomatous elements are encountered in testicular masses, anticipating a poorer prognosis and low five year survival rates, aggressive management to be given keeping in view of targeting sarcomatous cells also and this treatment is to be tailored individually till literature comes up with a standard treatment protocol guidelines as are existing with usually encountered testicular masses of carcinomatous origin.

\section{References}

1. Malagon HD, Valdez AM, Moran CA, et al. Germ cell tumours with sarcomatous components: a clinicopathologic and immunohistochemical study of 46 cases. Am J Surg Pathol 2007;31:1356-62.

2. Luke T. Lavallée, Nicolas LD Roustan Delatour etal. A unique case of a sarcoma arising in a testicular non-seminomatous mixed germ cell tumour with a predominant yolk sac component . Can Urol Assoc J 2011;5(1):E81-E83;

DOI:10.5489/cuaj.10069
3. Ulbright TM, Michael H, Loehrer PJ et al. Spindle cell tumours resected from male patients with germ cell tumours. A clinicopathologic study of 14 cases. Cancer 1990;65:148-56.

4. Brian Kelly, Dara Lundon etal Embryonal rhabdomyosarcoma of the testis. Can Urol Assoc J 2011;5(1):e7-e10; DOI:10.5489/cuaj.10047

5. Ambrose J. Hertzog Rhabdomyosarcoma Of The Testis Report OF Two CASES. amjcancer/28/1/131

6. A Korfel, L Fischer etal Testicular germ cell tumor with rhabdomyosarcoma successfully treated by disease-adapted chemotherapy including high-dose chemotherapy: case report and review of the literature. bmt/journal/v28/n8/full/1703212

7. Armita Bahrami, Jae Y. Ro, and Alberto G. Ayala (2007) An Overview of Testicular Germ Cell Tumors. Archives of Pathology \& Laboratory Medicine: August 2007, Vol. 131, No. 8, pp. 1267-1280.

8. Jaclyn C Milose Christopher P Filson etal Role of biochemical markers in testicular cancer: diagnosis, staging, and surveillance. oaju-4-001

9. Kabukcuoglu F, Sungun A, Senturk BA, et al. Mixed germ cell tumour of the ovary with sarcomatous component. Pathol Oncol Res 2001;7:60-2. 